\title{
Tumor Biological Aspects of Epithelial versus Mesenchymal Tumors of the Gastrointestinal Tract
}

\author{
Chandrajit P. Raut ${ }^{a, b, c}$ \\ a Department of Surgery, Brigham and Women's Hospital, Harvard Medical School, Boston, MA, USA; \\ ${ }^{b}$ Center for Sarcoma and Bone Oncology, Dana-Farber Cancer Institute, Boston, MA, USA; \\ ${ }^{\mathrm{c}}$ Harvard Medical School, Boston, MA, USA
}

\section{Keywords \\ Carcinoma - Sarcoma - Margins · Surgery . \\ Gastrointestinal tract $\cdot$ Stomach $\cdot$ Rectum}

\section{Summary}

One of the holy grails of cancer surgery for surgical oncologists is to perform a macroscopically complete resection of the affected organ or site with negative microscopic margins. The surgical oncologist must also be mindful of what constitutes appropriately-sized negative margins. However, what is necessary, based on the best available evidence, can vary considerably for different malignancies arising within the same organ. This review compares two different gastric and rectal malignancies, one each of epithelial and of mesenchymal origin. Data supporting extent of margins will be reviewed, illustrating the importance of knowing the histologic diagnosis prior to surgery to provide proper oncologic care.

(C) 2018 S. Karger GmbH, Freiburg

\section{Introduction}

Tumors of epithelial origin (carcinomas) and mesenchymal origin (sarcomas) can arise in the gastrointestinal (GI) tract. Within the body of literature of individual tumor types, prospective studies have provided some guidance for identifying optimal margins. Resulting guidelines are often based on experience with local and distant recurrence rates based on different margins of resection and are largely dependent on the inherent tumor biology of each type of malignancy. However, very little has been written contrasting the difference in surgical approach for tumors of such different embryonic origin.

To put this in focus, let us compare the multidisciplinary management of two pairs of scenarios: gastric adenocarcinoma versus gastric gastrointestinal tumor (GIST) and rectal carcinoma versus colorectal leiomyosarcoma. For gastric adenocarcinoma, the traditional dogma is to pursue a $5-\mathrm{cm}$ margin plus appropriate lymphadenectomy. For gastric GIST, contemporary data suggest that only a minimal negative margin, at most, is needed, and no lymphadenectomy is necessary. For rectal carcinoma, generally $1 \mathrm{~cm}$ or more of negative margin have been traditionally recommended, though specifications are changing. Mesorectal excision and lymphadenectomy is necessary. For colorectal/pelvic leiomyosarcoma, the radial margins into surrounding tissue are of greater concern, often necessitating en bloc resection of contiguous structures, but without need for lymphadenectomy.

This manuscript will explore the clinical data supporting current recommendations in these two pairs of scenarios and review the biological basis behind these guidelines. Four key questions will be addressed:

1) What constitutes a positive margin? Is it ink on tumor or ink less than $1 \mathrm{~mm}$ from tumor? What surfaces are evaluated?

2) What is an optimal margin distance? No ink at margin, greater than $1 \mathrm{~mm}, 1 \mathrm{~cm}, 5 \mathrm{~cm}$ ? What about biologic barriers such as fascia or bone?

3) Why are surgical margin considerations different for epithelial malignancies (carcinomas) and mesenchymal malignancies (sarcomas)?

4) What is the impact of a margin-positive (R1) resection in carcinomas versus sarcomas?

\section{KARGER}

(c) 2018 S. Karger GmbH, Freiburg
Chandrajit P. Raut, M.D., M.Sc. 
Fig. 1. Margin definitions in orthopedic oncology and surgical oncology

\begin{tabular}{|l|l|l|l|}
\hline Enneking/Orthopedic Oncology & all normal tissue of \\
compartment involved \\
encases specimen
\end{tabular}

\section{Definitions: Enneking Margins versus Surgical Oncology Resection Margins}

Due to inherent differences in the surgical approaches for bone and soft tissue sarcomas, resections of such sarcomas have been classified differently in the orthopedic oncology and surgical oncology literature. Orthopedic oncologic resections commonly use the Enneking classification [1] (fig. 1). Radical resections refer to operations in which all normal tissue of the compartment involved encases the specimen. Wide resections include nonreactive normal tissue along the entire periphery of the specimen. In marginal resections, the so-called 'pseudocapsule' forms the periphery of all or part of the specimen. In intralesional resections, the lesion itself forms the periphery of all or part of the specimen. In the surgical oncology literature, terminology used across all cancer types is applied for sarcoma resections. $R 0$ resections are macroscopically complete, with negative microscopic margins. $R 1$ resections are also macroscopically complete, but with positive microscopic margins. $R 2$ resections are macroscopically incomplete. Generally, ink on the tumor surface constitutes a positive margin. For sarcoma resections, margins that are less than $1 \mathrm{~mm}$ may often be considered positive as well. For the purposes of this review, extent of margin resection will utilize the conventions used in the surgical oncology literature.

\section{Sarcoma Margin Guidelines}

The US National Comprehensive Cancer Network (NCCN) and the European Society for Medical Oncology (ESMO) have developed guidelines for sarcoma management, which include commentary on margin. For both GIST and non-GIST sarcomas, the NCCN recommends obtaining oncologically appropriate guidelines (queried August 29, 2018) [2]. Close margins may be necessary to preserve critical structures such as vessels, nerves, and joints. However, there are no firm recommendations for ideal margin width. For GISTs in particular, re-resection is generally not indicated for microscopically positive margins on final pathology. For non-GIST sarcomas, a margin of at least $1 \mathrm{~cm}$ should be the goal, if possible. If margins are microscopically positive, re-resection should be considered.

The ESMO does not specify firm recommendation for margin width, but mentions considering anatomic barriers such as fascia or periosteum [3]. Furthermore, the ESMO guidelines recommend considering neoadjuvant therapies for close margins and making distinctions in extent of resection based on histologic subtype. The ESMO GIST guidelines recommend aiming for a macroscopically complete, margin-negative (R0) resection but macroscopically complete, margin-positive (R1) resections are reasonable to avoid potentially functional sequelae from what an R0 resection may entail [4].

The College of American Pathologists have published recommendations on reporting margins. They recommend ideally pursuing wide margins of at least $2 \mathrm{~cm}$ or more of normal tissue around all margins. For margins less than $2 \mathrm{~cm}$, location of those margins (superior, lateral, deep, etc.) should be specified as should the actual distance of tumor from margin.

In a single-institution series from the prospective surgical database from the Memorial Sloan Kettering Cancer Center (MSKCC), positive margin resections were associated with an increased risk of local recurrence and distant metastasis and a lower rate of diseasespecific survival [5]. Importantly, however, $72 \%$ of patients with positive margins from that series had no recurrence (local or distant).

\section{Why Should We Treat Margins Differently in Carcinomas versus Sarcomas?}

In general, carcinomas such as gastric or rectal adenocarcinoma are organ-based whereas most sarcomas tend to be cavity- or com- 
Table 1. Guidelines for gastric adenocarcinoma resection

\begin{tabular}{ll}
\hline Practice Guidelines & Size/localization \\
\hline $\begin{array}{r}\text { National Comprehensive } \\
\text { Cancer Network (US) }\end{array}$ & $>4 \mathrm{~cm}$ from gross \\
tumor
\end{tabular}

partment-based. Even when sarcomas arise from hollow viscera, such as gastric GIST or visceral leiomyosarcoma, the tumors may grow more variably in an exophytic or intraluminal direction. Carcinomas have traditionally been described as being more infiltrative while sarcomas are more expansile. Furthermore, carcinomas are more likely to spread initially through lymphatic channels rather than through the hematogenous route more commonly associated with sarcoma dissemination. This reflects a difference in tumor biology, and it has important implications in terms of the extent of surgery.

There are multiple types of surgical margins which have different relevance for different malignancies, as previously described in a paper discussing surgical margins for pancreatic cancer [6]. First, transected margins are those involving dissection or division across an organ, as with dividing across the pancreas body during a Whipple procedure. This margin is critical for carcinomas and for organ-based sarcomas. Second, dissected surfaces, i.e. those where there is division of continuous adventitia or lymphovascular channels, are again important for carcinomas. These include the surfaces around the periphery of an organ; in a Whipple procedure, these would include the anterior and posterior pancreatic margins (around the head and the uncinate process) and the superior mesenteric artery and vein margins. Third are the mobilized surfaces, those which require separation along a developed embryologic plane. For sarcomas, the third type of margin along mobilized surfaces is quite relevant. Here, there is separation along developed embryologic planes through which a sarcoma may extend in an exophytic manner; this is different from carcinomas but quite relevant in terms of risk of recurrence for sarcomas.

\section{Gastric Adenocarcinoma versus Gastric Gastrointestinal Stromal Tumor}

In the management of gastric adenocarcinomas, the ' $5 \mathrm{~cm}$ dogma' has served as the basis for justifying extent of resection. In the 1980s, margins of less than 3-6 cm from the gross tumor were associated with increased risk for R1 resection [7]. Therefore, based on this, the US NCCN recommended at least a $4 \mathrm{~cm}$ margin from the gross tumor. Japanese Gastric Cancer Treatment guidelines recommended a greater than $2 \mathrm{~cm}$ margin for T1 tumors and a $3-$ to 5-cm margin for T2-T4 tumors (table 1) [8, 9]. Studies demonstrated that an R0 resection was associated with better overall survival and disease-specific survival than R1 resection [10]. Amongst R0 patients, wider margins were associated with improved overall survival, most notably in T2 patients [11]. However, this was not
Table 2. Relative importance of different surgical margins for gastric malignancies

\begin{tabular}{lll}
\hline & $\begin{array}{l}\text { Gastric } \\
\text { adenocarcinoma }\end{array}$ & $\begin{array}{l}\text { Gastric gastrointestinal } \\
\text { stromal tumor }\end{array}$ \\
\hline Transection margin & ++++ & + \\
Dissection margin & ++++ & $-^{*}$ \\
Mobilization margin & - & $-{ }^{*}$ \\
\hline
\end{tabular}

${ }^{\star}$ No tumor disruption.

consistent across all T stages. Similarly, wider margins were associated with better outcomes for stage I, but there was no difference for stage II or stage III [12].

In contrast, margins can be much smaller for GISTs. Data combining the American College of Surgeons Oncology Group Z9000 and Z9001 studies demonstrated that patients who underwent an $\mathrm{R} 0$ versus $\mathrm{R} 1$ resection had no difference in recurrence-free survival regardless of whether or not they were treated with adjuvant imatinib [13].

Thus, a typical resection for gastric adenocarcinoma requires a total or distal gastrectomy or some other segmental gastrectomy, usually with $5-\mathrm{cm}$ margins, as well as a D2 lymphadenectomy. In contrast, for GIST, resection usually involves a partial wedge gastrectomy with simply negative margins (but not wide margins) and no lymphadenectomy. In terms of the potentially oncologicallyrelevant margins, the transection margin is critical for gastric cancer but less so for gastric GIST (table 2). Dissection margins including lymph nodes are important for gastric cancer but not necessarily so for gastric GIST other than maintaining a clean plane with no tumor disruption. Mobilization margins are again relatively less important for both, provided the specimen can be mobilized off of surrounding uninvolved tissue without tumor disruption.

\section{Rectal Adenocarcinoma versus Rectal/Pelvic Leiomyosarcoma}

Optimal margins for rectal adenocarcinoma are, generally, $5 \mathrm{~cm}$ proximally and $1 \mathrm{~cm}$ distally. Initially, $5-\mathrm{cm}$ distal margins were considered necessary [14], but data later confirmed that smaller distal margins were not associated with worse oncologic outcomes $[15,16]$. Margins of less than $1 \mathrm{~cm}$ may be acceptable following neoadjuvant chemoradiation therapy in an attempt to preserve the anal sphincter $[17,18]$. A total mesorectal excision is important and generally provides an adequate circumferential margin (usually greater than 1-2 mm from resected margin to tumor) [19]. Microscopic tumor deposits may be discontiguous and may be present in intramural and mesorectal planes up to $5 \mathrm{~cm}$ from the tumor site, thereby emphasizing the importance of that circumferential dissection [20].

In general, the impact of the size of the distal rectal margin on local recurrence, distant metastatic rates, and overall survival is uncertain. Most studies demonstrated no significant difference in 
Table 3. Relative importance of different surgical margins for rectal malignancies

\begin{tabular}{lll}
\hline & $\begin{array}{l}\text { Rectal } \\
\text { adenocarcinoma }\end{array}$ & $\begin{array}{l}\text { Rectal/pelvic } \\
\text { leiomyosarcoma }\end{array}$ \\
\hline Transection margin & $\begin{array}{ll}+++ \\
\text { rectal (proximal /distal) } \\
\text { margins }\end{array}$ & $\begin{array}{l}++++ \\
\text { if vascular origin }\end{array}$ \\
Dissection margin & ++++ & ++ \\
Mobilization margin & mesorectal margin & + \\
\hline
\end{tabular}

local recurrence with distal rectal margins greater than or less than $1 \mathrm{~cm}$, particularly in conjunction with neoadjuvant therapy [21]. In contrast, an inadequate circumferential margin (less than 1-2 mm) was associated with increased risk of local recurrence and a decreased disease-specific survival rate [21].

For visceral leiomyosarcomas, considerations are different. True visceral sarcomas, other than GIST, are generally exceedingly rare. Although primary rectal leiomyosarcomas may be rare, sarcomas may commonly arise in the pelvis. Different histologies may be involved, including well-differentiated liposarcoma, dedifferentiated liposarcoma, leiomyosarcoma, solitary fibrous tumor, and malignant peripheral nerve sheath tumors among others. Data from the MSKCC database suggested that there was no significant difference in disease-specific survival for retroperitoneal sarcomas after R0 versus R1 resection, and in general, considerations for pelvic sarcomas would be the same [22]. In truth, it is quite likely that there are few true R0 resections. However, there was a significant difference in disease-specific survival between patients who undergo R1 and R2 resection. Those that underwent an incomplete resection (R2) had survival rates that were only marginally better than patients with unresectable disease who did not have surgery.

Rates of local recurrence also depend significantly on histologic subtype. Amongst retroperitoneal and pelvic sarcomas, liposarcoma has a high risk of local recurrence, both well-differentiated and dedifferentiated [23]. In contrast, leiomyosarcomas generally have a low rate of local recurrence and are more likely to recur with distant metastases.

Drawing from this information, for visceral leiomyosarcomas such as rectal leiomyosarcomas, the goal of surgery is to ideally get a negative margin but not necessarily perform the same sort of dissection as for rectal adenocarcinoma. Although a total mesorectal excision is not generally necessary, it does provide a consistent and natural plane of dissection, particularly if the patient has received neoadjuvant radiation therapy; thus, it can be done in a manner similar to that for rectal adenocarcinoma. However, a lymphadenectomy is not indicated. All efforts at sphincter preservation are important, as is true for any rectal malignancy, but not to the point that margins are compromised. There are no specific guidelines for ideal margins for either the proximal or distal rectal margin.
Although sarcomas are generally considered 'pushing' tumors, they frequently demonstrate organ invasion, with resulting consequences on recurrence and survival. In a single-institution series, $59 \%$ of dedifferentiated liposarcomas and $52 \%$ of leiomyosarcomas arising in the retroperitoneum demonstrated histologic organ invasion into adjacent organs, and the presence of histologic organ invasion was associated with higher rates of distant recurrence and worse overall survival [24].

Transection margins are extremely important for rectal cancer, both proximally and distally (table 3 ). A negative margin is important for a rectal leiomyosarcoma, but no specific distance has been established. Dissection margins are critically important for rectal cancer, particularly the mesorectal excision margin. For rectal leiomyosarcomas, this is essentially a radial margin, and again a negative margin is ideal though often more challenging given the disruption of tissue planes. Mobilization margins are generally less critical for contained rectal adenocarcinoma though this may not be the case for patients with extension into the perirectal fat. Mobilization margins are important for visceral leiomyosarcomas, but again the predominant pattern of failures is distant recurrence and not necessarily local recurrence.

\section{Conclusions}

At present, there remains no consensus on optimal margins for most cancers. In general, for carcinomas, the transection and, to a lesser extent, dissection margins are quite relevant. Positive margins can usually lead to higher rates of local recurrence. Impact on the rates of distant metastasis and the overall survival are quite variable, though. For sarcomas, the dissection and mobilization margins are quite relevant, and to a lesser extent the transection margins. Positive margins were associated with higher rates of local recurrence and distant metastasis as well as reduced diseasespecific or overall survival for most sarcomas, though not necessarily GIST. Planned adjuvant/neoadjuvant therapy may reduce the risk for specific histologies and sites of origin. In general, for both epithelial and mesenchymal tumors, tumor biology is a critically important factor, and oncologic surgery should be planned accordingly.

Therefore, as we consider optimal margins for carcinomas versus sarcomas, one must take into account tumor histology and tumor biology, evidence-based guidelines for adequate or biologic margins, anatomic site, critical surrounding structures, and the use of neoadjuvant or adjuvant therapy.

\section{Disclosure Statement}

The author has no conflicts of interest to report. 


\section{References}

1 Enneking WF, Spanier SS, Goodman MA: A system for the surgical staging of musculoskeletal sarcoma. Clin Orthop Relat Res 1980;153:106-120.

2 von Mehren M, et al: Soft Tissue Sarcoma, Version 2.2018, NCCN Clinical Practice Guidelines in Oncology. J Natl Compr Canc Netw 2018;16:536-563.

3 Casali PG, et al: Soft tissue and visceral sarcomas: ESMO-EURACAN Clinical Practice Guidelines for diagnosis, treatment and follow-up. Ann Oncol 2018; DOI: 10.1093/annonc/mdy096.

4 Casali PG, et al: Gastrointestinal stromal tumours ESMO-EURACAN Clinical Practice Guidelines for diagnosis, treatment and follow-up. Ann Oncol 2018; DOI: 10.1093/annonc/mdy095.

5 Stojadinovic A, et al: Analysis of the prognostic significance of microscopic margins in 2,084 localized primary adult soft tissue sarcomas. Ann Surg 2002;235: 424-434.

6 Ethun CG, Kooby DA: The importance of surgical margins in pancreatic cancer. J Surg Oncol 2016;113 283-288.

7 Bozzetti F, et al: Adequacy of margins of resection in gastrectomy for cancer. Ann Surg 1982;196:685-690.

8 Postlewait LM, Maithel SK: The importance of surgical margins in gastric cancer. J Surg Oncol 2016;113:277282.

9 Japanese Gastric Cancer Association: Japanese gastric cancer treatment guidelines 2010 (version 3). Gastric Cancer 2011;14:113-123.
10 Hallissey MT, et al: Resection-line involvement in gastric cancer: a continuing problem. Br J Surg 1993;80: 1418-1420.

11 Barbour AP, et al: Adenocarcinoma of the gastroesophageal junction: influence of esophageal resection margin and operative approach on outcome. Ann Surg 2007;246:1-8.

12 Squires MH 3rd, et al: Is it time to abandon the $5-\mathrm{cm}$ margin rule during resection of distal gastric adenocarcinoma? A multi-institution study of the U.S. Gastric Cancer Collaborative. Ann Surg Oncol 2015;22: 1243-1251.

13 McCarter MD, et al: Microscopically positive margins for primary gastrointestinal stromal tumors: analysis of risk factors and tumor recurrence. J Am Coll Surg 2012;215:53-59; discussion 59-60.

14 Goligher JC, Dukes CE, Bussey HJ: Local recurrences after sphincter saving excisions for carcinoma of the rectum and rectosigmoid. Br J Surg 1951;39:199-211.

15 Williams NS, Dixon MF, Johnston D: Reappraisal of the 5 centimetre rule of distal excision for carcinoma of the rectum: a study of distal intramural spread and of patients' survival. Br J Surg 1983;70:150-154.

16 Paty PB, et al: Treatment of rectal cancer by low anterior resection with coloanal anastomosis. Ann Surg 1994;219:365-373.

17 Kuvshinoff B, et al: Distal margin requirements after preoperative chemoradiotherapy for distal rectal carcinomas: are $\leq 1 \mathrm{~cm}$ distal margins sufficient? Ann Surg Oncol 2001;8:163-169.
8 Moore HG, et al: Adequacy of 1-cm distal margin after restorative rectal cancer resection with sharp mesorectal excision and preoperative combined-modality therapy. Ann Surg Oncol 2003;10:80-85.

19 Nagtegaal ID, et al: Circumferential margin involvement is still an important predictor of local recurrence in rectal carcinoma: not one millimeter but two millimeters is the limit. Am J Surg Pathol 2002;26:350357.

20 Heald RJ, Husband EM, Ryall RD: The mesorectum in rectal cancer surgery - the clue to pelvic recurrence? $\mathrm{Br}$ J Surg 1982;69:613-616.

21 Mukkai Krishnamurty D, Wise PE: Importance of surgical margins in rectal cancer. J Surg Oncol 2016;113: 323-332.

22 Kirane A, Crago AM: The importance of surgical margins in retroperitoneal sarcoma. J Surg Oncol 2016; 113:270-276.

23 Gronchi A, et al: Variability in patterns of recurrence after resection of primary retroperitoneal sarcoma (RPS): a report on 1007 patients from the multi-institutional collaborative RPS working group. Ann Surg 2016;263:1002-1009.

24 Fairweather M, et al: Incidence and adverse prognostic implications of histopathologic organ invasion in primary retroperitoneal sarcoma. J Am Coll Surg 2017; 224:876-883. 